\title{
Physical Activity Patterns and Neighborhood Characteristics of First-Generation Latina Immigrants Living in Arizona: Cross-sectional Study
}

\author{
Rodney P Joseph ${ }^{1}, \mathrm{PhD}$; Sonia Vega-López ${ }^{2,3}, \mathrm{PhD} ;$ Seung Yong $\mathrm{Han}^{4}, \mathrm{PhD}$ \\ ${ }^{1}$ Center for Health Promotion and Disease Prevention, Edson College of Nursing and Health Innovation, Arizona State University, Phoenix, AZ, United \\ States \\ ${ }^{2}$ College of Health Solutions, Arizona State University, Phoenix, AZ, United States \\ ${ }^{3}$ Southwest Interdisciplinary Research Center, Arizona State University, Phoenix, AZ, United States \\ ${ }^{4}$ Edson College of Nursing and Health Innovation, Arizona State University, Phoenix, AZ, United States
}

Corresponding Author:

Rodney P Joseph, PhD

Center for Health Promotion and Disease Prevention

Edson College of Nursing and Health Innovation

Arizona State University

$500 \mathrm{~N} 3 \mathrm{rd} \mathrm{St}$

Phoenix, AZ, 85004

United States

Phone: 16024960772

Fax: 16024961128

Email: rodney.joseph@asu.edu

\section{Abstract}

Background: Metabolic diseases, including obesity and type 2 diabetes, are a major health concern for Latina immigrants. Performing regular aerobic physical activity (PA) is a lifestyle behavior associated with the prevention and control of these conditions. However, PA levels of most Latina immigrants are below national guidelines. Neighborhood environmental factors may influence the PA levels of adults, but limited research has explored associations between the neighborhood environment and PA levels among Latina immigrants.

Objective: The objective of this study was to explore the PA patterns of first-generation US Latina immigrants and how neighborhood environmental factors are related to those PA patterns.

Methods: Using a cross-sectional study design, 50 first-generation Latina immigrants completed the International Physical Activity Questionnaire (IPAQ) and the Neighborhood Scales Questionnaire, which assessed 6 perceived neighborhood factors: (1) walking environment, (2) aesthetic quality, (3) safety, (4) violence, (5) social cohesion, and (6) activities with neighbors. Median self-reported metabolic equivalent (MET)-minutes/week of PA were used to summarize domain-specific (ie, work, domestic/household, leisure, and transportation) and intensity-specific (ie, walking, moderate, vigorous, moderate to vigorous) PA patterns. Logistic regression examined associations between neighborhood factors and engaging in leisure-time PA (ie, dichotomous outcome of some versus no leisure-time PA), transportation PA (ie, dichotomous outcome of some versus no transportation PA), and meeting national PA guidelines (ie, dichotomous outcome of meeting versus not meeting guidelines).

Results: Preliminary analyses showed that 10 participants reported excessively high PA levels and 1 participant had incomplete PA data; these women were excluded from analyses based on IPAQ scoring guidelines. The remaining 39 participants (mean age 40.5 years; mean length of US residency 4.6 years) reported a median of 4512 MET-minutes/week of total PA. The majority of PA was acquired through domestic activities (median 2160 MET-minutes/week), followed by leisure-time PA (median 396 MET-minutes/week), transportation PA (median 198 MET-minutes/week), and work PA (0 MET-minutes/week). Intensity-specific PA patterns showed a median of 594 MET-minutes/week of walking activity and 3500 MET-minutes/week of moderate-to-vigorous PA. Logistic regression models indicated that the neighborhood factors of walking environment, aesthetic quality, and safety were positively associated with engaging in leisure-time PA (odds ratios of 5.95, 95\% CI 1.49-23.74; 2.45, 95\% CI 1.01-5.93; and 3.30, 95\% CI 1.26-8.67, respectively) and meeting national PA guidelines (odds ratios of 4.15, 95\% CI 1.13-15.18; 6.43, 
95\% CI 1.45-28.39; and 2.53, 95\% CI 1.00-6.36, respectively). The neighborhood factors of violence, social cohesion, and activities with neighbors were not significantly associated with PA outcomes.

Conclusions: Although most participants met national PA guidelines (ie, $\geq 500$ MET-minutes/week of moderate-to-vigorous PA), the majority of their PA was achieved through domestic activities, with limited leisure, transportation, and work PA. Given that leisure-time PA in particular plays a significant role in improving health outcomes, findings suggest that many Latina immigrants could benefit from a leisure-time PA intervention. Such interventions should consider neighborhood environmental influences, as these factors may serve as determinants of PA.

(JMIR Form Res 2021;5(5):e25663) doi: 10.2196/25663

\section{KEYWORDS}

emigrants and immigrants; physical activity; exercise; residence characteristics; female; metabolic disease; Latina; immigrants; emigrants; health outcomes

\section{Introduction}

Metabolic disease conditions are a major health concern for Latina immigrants. Findings from the Hispanic Community Health Study/Study of Latinos $(\mathrm{N}=16,415)$ indicate that $45 \%$ of first-generation Latina immigrants (ie, Latinas born outside of the United States) are obese [1] and 17\% have type 2 diabetes [2]. In comparison, national surveys estimate the prevalence of these conditions as $38 \%$ and $7 \%$, respectively, among non-Latina White women and $40 \%$ and $9 \%$ among the US population as a whole [3,4]. Important to the discussion on the prevalence of metabolic diseases among Latina immigrants is the pattern in which they develop. Upon initial entry into the United States, Latina immigrants have an equal-to-lower risk prevalence of obesity and diabetes compared with US-born Latinas and non-Latina Whites [5-7]. However, as duration of US residency increases, so does risk for developing obesity and diabetes conditions [2,5-10].

The high metabolic disease burden among Latina immigrants represents a major public health concern. Latinx immigrants are the largest immigrant group in the United States, accounting for approximately $40 \%$ of the total US immigrant population, and are projected to remain the largest immigrant population through at least the year 2055 [11]. Thus, a better understanding of lifestyle behaviors that may contribute to the high metabolic disease prevalence among first-generation Latina immigrants with longer duration of US residency is essential to developing interventions to address this public health concern.

Regular aerobic physical activity (PA) is an independent lifestyle behavior associated with the prevention and control of both obesity [12-14] and diabetes [15]. However, the PA levels of most Latina immigrants are below the national guidelines of 150 minutes/week of moderate-intensity PA [16-18]; data on this population are limited due to the limited number of studies that differentiate by generational status. Existing data also show a decline in PA as duration of US residency increases [19]. This decline in PA parallels the increase in metabolic disease risk observed among this population of US Latinas. Interventions designed to promote sustained high levels of PA may be key in addressing the metabolic disease prevalence among Latina immigrants.

The development of effective PA interventions requires researchers to have extensive understanding of the PA patterns of Latina immigrants as well as the social and environmental factors that may influence PA behaviors. The purpose of this study was to explore the PA patterns of first-generation Latina immigrants and the neighborhood environments in which they reside in the metropolitan area of Phoenix, Arizona. This research was conducted as part of a formative process to inform the development of culturally tailored PA interventions for first-generation Latina immigrants. First, we explored domain-specific PA engagement (ie, occupational, household, transportation, and leisure-time PA). Given the limited research on this topic among recently immigrated first-generation Latinas $[16,18,20]$ and emerging evidence suggesting that domain-specific PA differentially influences health outcomes (ie, high levels of leisure-time PA have the most profound impact on improving health outcomes [21-23], while high levels of occupational PA may have a limited-to-negative effect on improving health outcomes [21,24-26]), such research is necessary to gain an understanding of the PA patterns of recently immigrated US Latinas. Second, we examined the social and physical characteristics of the neighborhoods in which recently immigrated Latinas reside. We were interested in neighborhood characteristics because most people spend the majority of their nonworking time in or around their residential neighborhood. Thus, the neighborhood characteristics of Latina immigrants are likely to influence the types of PA promoted through a PA intervention $[16,18,20]$.

\section{Methods}

\section{Study Design, Setting, and Participant Recruitment}

A cross-sectional study design with self-report survey data was used. Data were collected as part of a broader study examining how immigration and integration experiences of recently immigrated first-generation Latinas influence perceptions of and opportunities for PA. Participants were a convenience sample of Latinas recruited from the metropolitan area of Phoenix. Recruitment strategies included flyer advertisements and in-person recruitment at predominately Hispanic-serving community outreach centers, health clinics, religious institutions, and local businesses. The Phoenix metropolitan area includes the cities of Phoenix, Mesa, and Chandler and is predominately comprised of urban and suburban neighborhoods. The region has an estimated 4.8 million residents, with $30 \%$ (ie, 1.5 million) identifying as Hispanic or Latinx [27]. Among residents identifying as Hispanic or Latinx, 400,000 are foreign-born and 
approximately 60,000 immigrated to the United States after 2010 [27]. Women were eligible for participation in this study if they (1) self-identified as Latina, (2) immigrated into the United States within the past 10 years, (3) were aged $\geq 18$ years, and (4) self-reported an ability to read in English or Spanish. Only women immigrating into the United States within the previous 10 years were included because the purpose of the larger study from which data were collected was to explore how contemporary social and contextual factors, including the impact of more restrictive immigration policies enacted during the US presidency of Donald Trump, were related to PA engagement among recently immigrated Latinas.

Women interested in study participation completed an eligibility screening interview either in-person or via telephone (according to the recruitment method). Eligible women were then given the option to complete the study questionnaire packet in English, Spanish, or a combined English/Spanish language using a completion method of their choice. Methods of survey completion included online via Qualtrics or by an oral interview. All participants were provided with an informed consent document prior to completing the survey. Participants completing the survey online provided informed consent by selecting the "continue" button on the webpage displaying the informed consent document that was accompanied by the following statement: "By clicking the Continue button, I acknowledge that I am at least 18 years old and that I consent to conducting the survey online." Verbal informed consent was obtained from participants completing the survey via the oral interview or pencil-and-paper format. After completing the survey, participants were provided a US \$25 gift card for study participation. All study procedures were approved by the Institutional Review Board of Arizona State University.

\section{Measures}

\section{Demographics}

Participant demographic characteristics were collected using a form developed for this study. Age, country of origin, and years of residence in the United States were obtained using the following open-ended questions: "How old are you?", "Where were you born?", and "How long have you been living in the United States?" Characteristics of primary language at home, monthly household income, education level, marital status, and employment status were asked using closed-ended questions modeled after items used in the 2017 Behavioral Risk Factor Surveillance System survey [28]. All demographic questions are available in Multimedia Appendix 1.

\section{Duration and Frequency of PA}

PA was assessed using the long version of the International Physical Activity Questionnaire (IPAQ) [29]. This 27-item questionnaire, previously validated in both English and Spanish $[29,30]$, asks respondents the duration (minutes/week) and frequency (days/week) of PA that they engage in according to 5 domains: (1) work, (2) transportation, (3) domestic/household, (4) leisure time, and (5) sitting. Responses were used to estimate the minutes/week that participants engaged in each activity. Self-reported minutes/week of activity were then weighted based on estimated energy expenditure (ie, metabolic equivalents
[METs]) to provide an estimate of total weekly PA volume (ie, MET-minutes/week), as well as an estimate of energy expenditure for time spent in domain- and intensity-specific (ie, walking, moderate, and vigorous intensity) activities. When respondents indicated that they did not perform activity in a given domain, the value for that domain was set to zero (eg, if a participant indicated that she did not perform paid or unpaid work outside of the home, the value for work-related PA was set to 0 ). All data were scored and reported according to guidelines published in 2005 [31] and were calculated as a continuous measure of MET-minutes/week.

\section{Neighborhood Environment}

The neighborhood environmental factors of walking environment, aesthetic quality, safety, violence, social cohesion, and activities with neighbors were assessed using the Neighborhood Scales Questionnaire [32]. Scales measuring neighborhood walking environment (7 items), aesthetic quality (5 items), safety (3 items), and social cohesion (4 items) have respondents rate their agreement with various statements using a 5-point Likert scale (ie, 1=strongly agree, 2=agree, 3=neutral, 4=disagree, and 5=strongly disagree). Example statements include "I often see other people walking in my neighborhood" (from the walking environment scale) and "I feel safe walking in my neighborhood, day or night" (from the safety scale). Scales assessing neighborhood violence (4 items) and activities with neighbors ( 4 items) have participants rate the frequency of specific events on a 4-point Likert scale (1=often, $2=$ sometimes, $3=$ rarely, and $4=$ never). Example items from these scales include "During the past 6 months, how often was there a fight in your neighborhood in which a weapon was used?" (from the violence scale) and "How often do you and other people in your neighborhood visit in each other's homes or speak with each other on the street?" (from the "activities with neighbors" scale). All scales were scored individually by calculating the mean score of individual scale items. For ease of data interpretation, scores were reverse ordered, when appropriate, to indicate that higher scores are associated with higher walkability, aesthetic quality, safety, social cohesion, violence, and activities with neighbors. The Neighborhood Scales Questionnaire was developed and validated in both English and Spanish [32] and has established test-retest reliability (ie, intraclass correlation coefficients ranging from .60 to .88) [32]. Internal consistency estimates (ie, Cronbach alpha coefficients) for the scales ranged from .78 to .92 in this study, which are comparable to previous research $[32,33]$.

\section{Statistical Analysis}

Descriptive statistics (ie, means, 95\% confidence intervals, medians, interquartile ranges, and frequencies) were used to summarize participant demographic, PA, and neighborhood variables. A series of regression models were used to examine associations between neighborhood environmental factors and the PA outcomes of leisure-time PA, transportation PA, and overall moderate-to-vigorous PA. Associations between neighborhood factors and work and household PA were not examined because we lacked a theoretical rationale for why neighborhood factors would influence these PA variables. Ordinal least squares (OLS) regression models, controlling for 
age and level of education, were used to examine the independent effect of each neighborhood factor on continuous PA outcomes in MET-minutes/week. Logistic regression, controlling for age, was used to examine associations between neighborhood factors and dichotomous PA outcomes of engaging in leisure-time PA (ie, engaging in 0 versus $>0$ MET-minutes/week of leisure-time PA), transportation PA (ie, engaging in 0 versus $>0$ MET-minutes/week of transportation PA), and meeting national PA guidelines (ie, engaging in $<500$ versus $\geq 500$ MET-minutes/week of moderate-to-vigorous PA). Level of education as a control variable was not included in logistic models because of perfect prediction. As a result of the collinearity of neighborhood variables, associations between each neighborhood variable (ie, walking environment, aesthetic quality, safety, violence, social cohesion, and activities with neighbors) and PA outcomes were examined separately. Stata/SE version 16.0 (StataCorp) was used for data analysis.

\section{Results}

\section{Participants}

A total of 50 first-generation Latinas participated in the study. However, preliminary data cleaning revealed that 10 participants reported unreasonably high PA data (ie, the sum of all walking, moderate, and vigorous PA time was greater than 960 minutes or 16 hours/day) and 1 participant had incomplete IPAQ data. These women were excluded from data analysis according to IPAQ scoring guidelines [31], resulting in a final sample size of 39 participants. Sensitivity analyses (ie, chi-square test for categorical variables and $t$ test for age and duration of US residency) were conducted to explore demographic differences between participants excluded from outcome analyses $(n=11)$ and those included. Results showed that the women excluded from the study had a lower education level than those included $(P=.003)$. No other demographic differences were observed between women included in the study and those excluded.

Among the 39 women included in outcome analyses, the mean age was 40.5 (SD 4.3) years and the mean duration of US residence was 4.6 (SD 1.0) years. The majority of the participants were from Mexico (27/39, 69\%), with the remaining participants from various Central and South America countries. Approximately half $(22 / 39,56 \%)$ of the women were married and the majority spoke Spanish exclusively at home (32/39, $82 \%)$. Based on data provided by the IPAQ, only $7(18 \%)$ of the 39 participants reported performing paid or unpaid work outside of the home. Complete demographic characteristics are presented in Table 1. 
Table 1. Demographic characteristics of study participants included in the outcome analysis $(\mathrm{N}=39)$.

\begin{tabular}{ll}
\hline Characteristic & Value \\
\hline Age (years), mean (95\% CI) & $40.5(36.2-44.8)$ \\
Age (years), median (minimum; maximum) & $39.0(2.4 ; 78.0)$ \\
Duration in the United States (months), mean (95\% CI) & $55.6(43.5-67.7)$ \\
Duration in the United States (months), median (minimum; maximum) & $52.0(5.0 ; 128.0)$ \\
Country of origin, $\mathbf{n}(\%)$ & $27(69)$ \\
$\quad$ Mexico & $5(13)$ \\
$\quad$ Venezuela & $4(10)$ \\
$\quad$ Colombia & $1(3)$ \\
Cuba & $1(3)$ \\
Dominican Republic & $1(3)$ \\
Nicaragua &
\end{tabular}

Primary language spoken at home, n (\%)

English

English and Spanish

Spanish

Monthly household income, $\mathrm{n}(\%)$

$\leq \$ 1000$

$\$ 1001-\$ 2000$

$\$ 2001-\$ 3000$

$\$ 3001-\$ 4000$

$>\$ 4000$

Don't know

Refused to respond

Education, n (\%)

Elementary

Middle school

High school

Some college or university

University graduate or postgraduate

Marital status, n (\%)

Single or no partner

Married

Cohabiting

Separated

Divorced

Widowed

Employment, n (\%)

Unemployed/looking for work

Unemployed/not looking for work

Homemaker

Student

Retired 


\begin{tabular}{cll}
\hline Characteristic & Value \\
\hline Other & $2(5)$ \\
Missing & $18(46)$ \\
\hline
\end{tabular}

\section{PA Outcomes}

Table 2 provides a detailed overview of intensity- and domain-specific PA patterns of our sample. Participants reported a median of 4512 MET-minutes/week of total PA. Intensity-specific PA outcomes showed that the majority of this PA was achieved through moderate-intensity activity (median
2820 MET-minutes/week). Median self-reported MET-minutes/week of walking and vigorous-intensity PA were 594 and 0, respectively. Overall, 85\% (33/39) of participants reported PA levels that met the requirements of the PA guidelines (ie, $\geq 500 \quad$ MET-minutes/week of moderate-to-vigorous PA).

Table 2. Summary statistics of intensity- and domain-specific metabolic equivalent (MET)-minutes/week of physical activity (PA).

\begin{tabular}{|c|c|c|c|}
\hline \multirow[b]{2}{*}{ Outcome } & \multirow[b]{2}{*}{ Participants, n (\%) } & \multicolumn{2}{|c|}{ MET-minutes/week of PA } \\
\hline & & Mean $(95 \% \mathrm{CI})$ & Median (IQR) \\
\hline Total $\mathrm{PA}^{\mathrm{a}}$ & $39(100)$ & $6128(4277-7979)$ & $4512(7271)$ \\
\hline \multicolumn{4}{|l|}{ PA by intensity } \\
\hline Walking & $39(100)$ & $963(532-1394)$ & $594(1386)$ \\
\hline Moderate & $39(100)$ & $3842(2696-4988)$ & $2820(4410)$ \\
\hline Vigorous & $39(100)$ & $1323(545-2101)$ & $0(1440)$ \\
\hline Moderate to vigorous ${ }^{b}$ & $39(100)$ & $5165(3456-6874)$ & $3500(6060)$ \\
\hline \multicolumn{4}{|l|}{ Achieved PA guidelines ${ }^{c}$} \\
\hline Met PA guidelines & $33(85)$ & $236(18-454)$ & $270(396)$ \\
\hline Did not meet PA guidelines & $6(15)$ & $7199(5225-9174)$ & $5040(7778)$ \\
\hline \multicolumn{4}{|l|}{ Work PA } \\
\hline Participants reporting working outside of the home & $7(18)$ & $6009(1171-10,846)$ & $4590(10,536)$ \\
\hline Work PA—full sample ${ }^{\mathrm{d}}$ & $39(100)$ & $1079(65-2092)$ & $0(0)$ \\
\hline \multicolumn{4}{|l|}{ Transportation PA } \\
\hline Participants reporting transportation PA & $23(59)$ & $584(365-803)$ & $360(594)$ \\
\hline Transportation PA—full sample ${ }^{\mathrm{d}}$ & $39(100)$ & $345(188-501)$ & $198(453)$ \\
\hline \multicolumn{4}{|l|}{ Domestic/household PA } \\
\hline Participations reporting domestic/household PA & $34(87)$ & $3514(2497-4531)$ & $2850(4024)$ \\
\hline Domestic/household PA—full sample ${ }^{\mathrm{d}}$ & $39(100)$ & $3063(2102-4025)$ & $2160(4560)$ \\
\hline \multicolumn{4}{|l|}{ Leisure-time PA } \\
\hline Participants reporting engaging in leisure-time PA & $26(67)$ & $24623(1146-3779)$ & $1042(2997)$ \\
\hline Leisure-time PA-full sample ${ }^{\mathrm{d}}$ & $39(100)$ & $1642(704-2580)$ & $396(1980)$ \\
\hline \multicolumn{4}{|l|}{ Sitting time } \\
\hline Weekday total & $38(97)$ & $333(267-399)$ & $300(240)$ \\
\hline Weekend total & 39 (100) & $327(250-405)$ & $240(240)$ \\
\hline
\end{tabular}

\footnotetext{
${ }^{\mathrm{a}}$ The sum of walking and moderate and vigorous PA, which is equal to the sum of work, transportation, domestic/household, and leisure-time PA domains.

${ }^{\mathrm{b}}$ The sum of moderate and vigorous PA.

${ }^{\mathrm{c}}$ Achieving PA guidelines was defined as engaging in $\geq 500$ MET-minutes/week of moderate-to-vigorous PA; not meeting PA guidelines was defined as engaging in $<500$ MET-minutes/week of moderate-to-vigorous PA.

${ }^{\mathrm{d}}$ Participants reporting that they did not engage in a domain-specific activity had their value for that given domain set to zero; full-sample values for each domain include these participants with their zero values and thus provide an overall summary statistic for the domain that includes all study participants.
} 
Examination of domain-specific PA patterns showed that domestic/household PA was the most frequent type of PA reported by participants $(34 / 39,87 \%)$ and, on average, how participants acquired the majority of their activity (ie, median 2160 MET-minutes/week). Leisure-time PA was the second most frequently reported domain of activity $(26 / 39,67 \%)$, followed by transportation $(23 / 39,59 \%)$ and work $(7 / 39,18 \%)$ PA. Additionally, although only 7 participants reported working outside of the home according to the IPAQ, the amount of PA acquired by these participants through work activity was higher than any other domain (4590 MET-minutes/week; see Table 2).

\section{Associations Between Neighborhood Environmental Factors and PA}

Table 3 shows the descriptive values for scales assessing neighborhood environmental factors. OLS regression results, presented in Table 4, revealed no significant associations between neighborhood environmental factors and continuous PA measures. Table 5 presents logistic regression models examining associations between neighborhood environmental factors and dichotomous PA outcomes of engaging in transportation PA versus not engaging in transportation PA, engaging in leisure-time PA versus not engaging in leisure-time PA, and meeting PA guidelines versus not meeting PA guidelines. Results showed that the neighborhood factors of walking environment, aesthetic quality, and safety were significantly associated with engaging in leisure-time PA (OR $5.95,95 \%$ CI 1.49 to 23.74 ; OR 2.45, $95 \%$ CI 1.01 to 5.93 ; and OR $3.30,95 \%$ CI 1.26 to 8.67 , respectively) and meeting national PA guidelines (OR 4.15, 95\% CI 1.13 to 15.18; OR $6.43,95 \%$ CI 1.15 to 28.39 ; and OR $2.53,95 \%$ CI 1.00 to 6.36 , respectively). No other significant associations were found.

Table 3. Summary statistics of perceived neighborhood factors.

\begin{tabular}{llll}
\hline & \multicolumn{2}{l}{ Neighborhood Scales Questionnaire [32] scale scores } \\
Perceived neighborhood factor & Observations, $\mathrm{n}$ & Mean (95\% CI) & \multicolumn{2}{l}{ Median (minimum; maximum) } \\
\hline Walking environment $^{\mathrm{a}}$ & 38 & $3.9(3.6-4.1)$ & $3.9(1.9 ; 5.0)$ \\
Aesthetic quality $^{\mathrm{a}}$ & 39 & $3.7(3.4-4.0)$ & $3.8(1.0 ; 5.0)$ \\
Safety $^{\mathrm{a}}$ & 38 & $3.4(3.1-3.7)$ & $3.7(1.0 ; 5.0)$ \\
Social cohesion $^{\mathrm{a}}$ & 35 & $3.1(2.8-3.4)$ & $3.3(1.0 ; 5.0)$ \\
Violence $^{\mathrm{b}}$ & 33 & $1.4(1.2-1.7)$ & $1.0(1.0 ; 5.0)$ \\
Activities with neighbors $^{\mathrm{b}}$ & 38 & $1.7(1.5-1.9)$ & $1.6(1.0 ; 3.4)$ \\
\hline
\end{tabular}

${ }^{\mathrm{a}} \mathrm{Score}$ range of 1 to 5 .

${ }^{\mathrm{b}}$ Score range of 1 to 4 . 
Table 4. Ordinal least squares (OLS) regression analyses examining associations between neighborhood factors and dichotomous physical activity (PA) outcomes.

OLS regression results for continuous outcomes ${ }^{\mathrm{a}}$

PA outcome and neighborhood factor

Observations, $\mathrm{n}$

Coefficient $(95 \% \mathrm{CI})$

PA domain: transportation

Walking environment

Aesthetic quality

Safety

Social cohesion

Violence

Activities with neighbors

PA domain: leisure

Walking environment

Aesthetic quality

Safety

Social cohesion

Violence

Activities with neighbors

PA intensity level: moderate to vigorous

Walking environment

Aesthetic quality

Safety

Social cohesion

Violence

Activities with neighbors

$$
\begin{aligned}
& -31.45(-217.21 \text { to } 154.30) \\
& -5.99(-161.78 \text { to } 149.79) \\
& 47.58(-91.97 \text { to } 187.13) \\
& -32.95(-194.04 \text { to } 128.15) \\
& 20.30(-156.13 \text { to } 196.73) \\
& 102.70(-97.72 \text { to } 303.12)
\end{aligned}
$$

734.54 (-595.48 to 2064.57$)$

558.27 ( -538.28 to 1654.81$)$

724.93 ( -266.29 to 1716.14$)$

189.49 (-819.58 to 1198.57$)$

-1201.72 (-2537.39 to 133.95$)$

-348.37 (-1778.59 to 1081.85$)$

1934.56 ( -418.61 to 4287.73$)$

1662.83 ( -273.18 to 3598.83 )

1168.56 (-634.70 to 2971.83 )

593.57 ( -1386.55 to 2573.70$)$

-1679.53 (-4229.44 to 870.37$)$

509.87 ( -2052.37 to 3072.10$)$

${ }^{\mathrm{a}}$ Controlled for age and level of education. 
Table 5. Logistic regression analyses examining associations between neighborhood factors and dichotomous physical activity (PA) outcomes. ${ }^{\text {a,b }}$

\begin{tabular}{|c|c|c|}
\hline PA outcome and neighborhood factor & Frequency, n (\%) & OR $(95 \% \mathrm{CI})$ \\
\hline \multicolumn{3}{|l|}{ PA domain: transportation } \\
\hline Did not engage in transportation PA & $16(41)$ & 1.00 \\
\hline Engaged in transportation PA & $23(59)$ & \\
\hline Walking environment & & $1.60(0.61-4.23)$ \\
\hline Aesthetic quality & & $0.83(0.38-1.78)$ \\
\hline Safety & & $1.22(0.60-2.47)$ \\
\hline Social cohesion & & $1.45(0.66-3.16)$ \\
\hline Violence & & $1.17(0.46-2.99)$ \\
\hline Activities with neighbors & & $1.75(0.59-5.16)$ \\
\hline \multicolumn{3}{|l|}{ PA domain: leisure-time } \\
\hline Did not engage in leisure-time PA & $13(33)$ & 1.00 \\
\hline Engaged in leisure-time PA & $26(67)$ & \\
\hline Walking environment & & $5.95 *(1.49-23.74)$ \\
\hline Aesthetic quality & & $2.45^{*}(1.01-5.93)$ \\
\hline Safety & & $3.30 *(1.26-8.67)$ \\
\hline Social cohesion & & $1.92(0.81-4.55)$ \\
\hline Violence & & $0.38(0.13-1.10)$ \\
\hline Activities with neighbors & & $0.67(0.24-1.83)$ \\
\hline \multicolumn{3}{|l|}{ Achieved national PA guidelines } \\
\hline Did not meet PA guidelines & $7(18)$ & 1.00 \\
\hline Met PA guidelines & $32(82)$ & \\
\hline Walking environment & & $4.15^{*}(1.13-15.18)$ \\
\hline Aesthetic quality & & $6.43 *(1.45-28.39)$ \\
\hline Safety & & $2.53 *(1.00-6.36)$ \\
\hline Social cohesion & & $1.29(0.53-3.15)$ \\
\hline Violence & & $0.43(0.16-1.20)$ \\
\hline Activities with neighbors & & $0.99(0.24-4.02)$ \\
\hline
\end{tabular}

${ }^{\mathrm{a}}$ Controlled for age.

${ }^{\mathrm{b}} \mathrm{PA}$ outcome variables for logistic regression analyses included engaging in transportation PA (ie, $>0$ metabolic equivalent [MET]-minutes/week of transportation PA) versus not engaging in transportation PA (0 MET-minutes/week of transportation PA), engaging in leisure-time PA (ie, >0 MET-minutes/week of leisure-time PA) versus not engaging in leisure-time PA (ie, 0 MET-minutes/week), and meeting national PA guidelines (ie, $\geq 500$ MET-minutes/week of moderate-to-vigorous PA) versus not meeting national PA guidelines (ie, <500 MET-minutes/week of moderate-to-vigorous PA).

*Significant at a $P$ value $<.05$.

\section{Discussion}

\section{Principal Findings}

This study explored PA patterns among first-generation Latina immigrants residing in the metropolitan area of Phoenix, Arizona, and the influence of perceived neighborhood environmental factors on these PA patterns. This work adds to the limited body of research on domain-specific PA patterns among first-generation Latina immigrants, with the majority of previous PA studies among Latinas having focused exclusively on leisure-time PA [34] and failing to differentiate by generational status. Given that first-generation immigrants likely have different lived experiences and lifestyle activities than Latinas who were born in the United States, work is needed in order to inform the development of PA interventions for this population.

Results showed that the majority of our sample (85\%) reported PA levels that met or exceeded national PA guidelines. Domain-specific outcomes indicated that participants accrued the majority of their PA through domestic and household activities, with limited PA performed for leisure or transportation purposes. The perceived neighborhood environmental factors of walking environment, aesthetic quality, and safety were positively associated with engaging in leisure-time PA and 
meeting national PA guidelines. The neighborhood factors of violence, social cohesion, and activities with neighbors were not related to PA outcomes.

\section{Comparisons With Prior Work}

Our finding that domestic and household activities accounted for the majority of PA performed reflects the outcomes of several previous studies examining PA patterns among Latinas $[8,20]$ and supports the notion that Latinas, regardless of generational status, perform extensive caretaking and household activities as part of their daily routine. Likewise, low levels of leisure-time PA and a limited number of participants engaging in paid or unpaid work outside the home have also been previously reported among studies comprised predominantly of first-generation Latinas [19,20,33]. Leisure-time PA, in particular, has profound benefits for reducing cardiometabolic disease conditions [23,35,36]. Moreover, when compared with other PA domains (ie, work and transportation), leisure-time PA may be more amendable to change in the context of a PA intervention because it is within the volitional control of many Latinas. In future work, researchers should explore intervention strategies to increase leisure-time PA among first-generation Latinas. Such work may be key to reducing the disproportionate metabolic disease burden in this high-risk population.

Our examination of overall and intensity-specific PA patterns showed that our sample reported engaging in rather high levels of overall PA (ie, 4512 MET-minutes/week), with the majority of the PA being at a moderate intensity. We speculate that these high PA levels may be a result of overreporting, as this is a commonly reported occurrence with self-report PA measures [37]. Nicaise and colleagues [20] illustrated this issue in a previous study with low-income Latinas using the IPAQ and accelerometers. When assessing PA levels using the IPAQ, the authors found that $73 \%$ of Latinas met PA guidelines, with the bulk of the PA being accrued through domestic or household activities [20]—similar to the outcomes of our study. However, when examining PA levels with accelerometers, this percentage was reduced to $20 \%$, suggesting that participants likely overestimated the intensity and the amount of PA accrued through domestic and household activities [20]. We speculate that a similar phenomenon may have impacted our study outcomes. Another key finding of our study was that our sample of Latinas reported limited vigorous-intensity PA, which also mirrors the outcome of several previous studies [18,20]. We hypothesize that this outcome was related to the limited amount of leisure-time PA reported by our sample, as vigorous PA is predominately achieved through purposeful exercise as opposed to daily activities [38].

When examining associations between neighborhood environmental factors and PA outcomes, linear regression models failed to show any significant associations. This might be due to the nonlinear patterns of the associations and a low statistical power due to the small sample size. However, logistic regression models revealed that the neighborhood factors of walking environment, aesthetic quality, and safety were associated with reported engagement in leisure-time PA and meeting national PA guidelines. These findings confirm previous studies showing that these factors are positively associated with
PA engagement [19,39-42]. We also found it interesting that these 3 factors are ones that recent immigrants likely have limited control over (ie, these factors are primarily driven by public policies and allocation of local government funds). This outcome highlights the importance of considering all levels of the social ecological model, including public policy and urban design planning, when developing PA promotion interventions.

The lack of significant associations between the 6 neighborhood environmental factors and engagement in some versus no transportation PA in logistic models may be related to transportation PA being a necessity for daily activities rather an option (ie, participants may have had no other type of transportation). However, we did not include survey items asking participants about their primary mode of transportation, which is needed to draw a more precise conclusion on this outcome. Likewise, the lack of associations between social cohesion, violence, and activities with neighbors and PA was reported previously in a study among Latinx residing in Massachusetts [40], suggesting that these 3 factors may not be key determinants of PA engagement among Latinas. Future studies with larger sample sizes are needed before definitive conclusions can be drawn.

\section{Limitations of the Study}

Limitations of our study include the use of a relatively small sample from a single metropolitan geographic region. The sample size was determined based on available funds, and the geographic region chosen was based on the population that the subsequent intervention would be developed to target. The small sample limited our ability to conduct additional subgroup analyses to further examine associations among duration of US residency (ie, $\leq 5$ years versus $>5$ years), neighborhood environmental factors, and PA. Further examination of neighborhood characteristics and PA patterns based on duration of residency status in the United States would be interesting to explore in future work. Likewise, given that our sample comprised women residing in urban and suburban areas of metropolitan Phoenix, the study findings should not be generalized to residents residing in rural areas. Additional research with a larger, more geographically diverse sample is warranted to confirm and expand on our findings. Another limitation was that we relied exclusively on self-report measures to assess PA and neighborhood characteristics. Including an objective PA measure would have provided further context regarding the PA patterns of our sample. However, this was not possible because of the limited resources of the study. Likewise, preliminary data analyses resulted in 11 women being excluded from the study based on IPAQ scoring guidelines for either reporting nonplausible PA data $(n=10)$ or providing incomplete data $(n=1)$. Sensitivity analyses showed that these participants reported lower education levels than participants who provided valid data. This suggests that the IPAQ may not be appropriate for Latinas of lower education levels, further limiting generalization of the study findings. Additionally, perceived neighborhood characteristics may differ from objectively measured characteristics (eg, neighborhood characteristics measured using geographic information systems, local crime/violence data). Future research among first-generation Latinas should consider the use of both subjective and objective 
PA measures and neighborhood measures, as both types of measures provide important insights into the PA patterns and neighborhood characteristics of Latinas. A final limitation of this study is that it did not include items assessing BMI or health status. Such information would have provided additional insight on the generalizability of the study findings.

\section{Strengths of the Study}

Despite its limitations, the current study has several strengths. First, this is one of few studies to examine domain-specific PA patterns among first-generation Latina immigrants, as most PA studies among Latinas have only included a measure of leisure-time PA [34] and failed to differentiate by generational status. Another strength of the study is that our sample was comprised of women from diverse countries of origin (ie, 69\% from Mexico and the remainder from various Central and South American countries). The composition of our sample likely reflects the changing Latinx immigrant population in the United
States (ie, immigration from Central and South American countries has increased in recent years, with immigration from Mexico slowly declining) [11].

\section{Conclusions}

Findings suggest that many first-generation Latinas could benefit from a leisure-time PA intervention. Given that leisure-time PA has pronounced benefits for promoting positive health outcomes, intervention efforts targeting leisure-time PA may be an effective method for researchers and public health professionals to reduce obesity and diabetes health disparities among Latina immigrants. Such interventions should consider the neighborhood environments in which first-generation Latinas reside, as these factors will likely influence the types of PA promoted in an intervention. Results of this study will be used to inform development of a culturally tailored PA intervention for the reduction of metabolic disease risk among first-generation Latina immigrants.

\section{Conflicts of Interest}

None declared.

\section{Multimedia Appendix 1}

Demographic questionnaire.

[DOCX File, 14 KB-Multimedia Appendix 1]

\section{References}

1. Kaplan RC, Avilés-Santa ML, Parrinello CM, Hanna DB, Jung M, Castañeda SF, et al. Body mass index, sex, and cardiovascular disease risk factors among Hispanic/Latino adults: Hispanic community health study/study of Latinos. J Am Heart Assoc 2014 Jul 09;3(4):e000923 [FREE Full text] [doi: 10.1161/JAHA.114.000923] [Medline: 25008353]

2. Schneiderman N, Llabre M, Cowie CC, Barnhart J, Carnethon M, Gallo LC, et al. Prevalence of diabetes among Hispanics/Latinos from diverse backgrounds: the Hispanic Community Health Study/Study of Latinos (HCHS/SOL). Diabetes Care 2014 Aug;37(8):2233-2239 [FREE Full text] [doi: 10.2337/dc13-2939] [Medline: 25061138]

3. Hales C, Carroll M, Fryar CD, Ogden CL. Prevalence of Obesity Among Adults and Youth: United States, $2015-2016$. NCHS Data Brief 2017 Oct(288):1-8 [FREE Full text] [Medline: 29155689]

4. Centers for Disease Control and Prevention, National Center for Chronic Disease Prevention and Health Promotion, Division of Diabetes Translation. National Diabetes Statistics Report, 2017: Estimates of Diabetes and Its Burden in the United States. 2017. URL: https://dev.diabetes.org/sites/default/files/2019-06/cdc-statistics-report-2017.pdf [accessed 2021-04-25]

5. Goel MS, McCarthy EP, Phillips RS, Wee CC. Obesity among US immigrant subgroups by duration of residence. JAMA 2004 Dec 15;292(23):2860-2867. [doi: $10.1001 /$ jama.292.23.2860] [Medline: 15598917 ]

6. Isasi CR, Ayala GX, Sotres-Alvarez D, Madanat H, Penedo F, Loria CM, et al. Is acculturation related to obesity in Hispanic/Latino adults? Results from the Hispanic community health study/study of Latinos. J Obes 2015;2015:186276 [FREE Full text] [doi: 10.1155/2015/186276] [Medline: 25893114]

7. Koya DL, Egede LE. Association between length of residence and cardiovascular disease risk factors among an ethnically diverse group of United States immigrants. J Gen Intern Med 2007 Jun;22(6):841-846 [FREE Full text] [doi: 10.1007/s11606-007-0163-y] [Medline: 17503110]

8. Wolin KY, Colangelo LA, Chiu BC, Gapstur SM. Obesity and immigration among Latina women. J Immigr Minor Health 2009 Oct;11(5):428-431 [FREE Full text] [doi: 10.1007/s10903-007-9115-1] [Medline: 18183486]

9. Oza-Frank R, Stephenson R, Narayan KMV. Diabetes prevalence by length of residence among US immigrants. J Immigr Minor Health 2011 Feb;13(1):1-8. [doi: 10.1007/s10903-009-9283-2] [Medline: 19688263]

10. Oza-Frank R, Cunningham SA. The weight of US residence among immigrants: a systematic review. Obes Rev 2010 Apr;11(4):271-280. [doi: 10.1111/j.1467-789X.2009.00610.x] [Medline: 19538440]

11. Budiman A. Key findings about U.S. immigrants. Pew Research Center. 2020 Aug 20. URL: https://www.pewresearch.org/ fact-tank/2019/06/17/key-findings-about-u-s-immigrants/ [accessed 2021-04-22]

12. Swift DL, Johannsen NM, Lavie CJ, Earnest CP, Church TS. The role of exercise and physical activity in weight loss and maintenance. Prog Cardiovasc Dis 2014;56(4):441-447 [FREE Full text] [doi: 10.1016/j.pcad.2013.09.012] [Medline: 24438736] 
13. Jakicic JM, Davis KK. Obesity and physical activity. Psychiatr Clin North Am 2011 Dec;34(4):829-840. [doi: 10.1016/j.psc.2011.08.009] [Medline: 22098807]

14. Jakicic J. Physical activity and weight loss. Nestle Nutr Inst Workshop Ser 2012;73:21-36. [doi: 10.1159/000341283] [Medline: 23128763]

15. Colberg SR, Sigal RJ, Fernhall B, Regensteiner JG, Blissmer BJ, Rubin RR, American College of Sports Medicine, American Diabetes Association. Exercise and type 2 diabetes: the American College of Sports Medicine and the American Diabetes Association: joint position statement executive summary. Diabetes Care 2010 Dec;33(12):2692-2696 [FREE Full text] [doi: $10.2337 / \mathrm{dc} 10-1548]$ [Medline: 21115771]

16. Sweatt SK, Willig AL, Agne AA, Powell JL, Cherrington AL. Physical Activity Patterns of Latina Immigrants Living in Alabama. J Racial Ethn Health Disparities 2015 Sep;2(3):365-372 [FREE Full text] [doi: 10.1007/s40615-015-0083-1] [Medline: 26413457]

17. Arredondo EM, Sotres-Alvarez D, Stoutenberg M, Davis SM, Crespo NC, Carnethon MR, et al. Physical Activity Levels in U.S. Latino/Hispanic Adults: Results From the Hispanic Community Health Study/Study of Latinos. Am J Prev Med 2016 Apr;50(4):500-508 [FREE Full text] [doi: 10.1016/j.amepre.2015.08.029] [Medline: 26597505]

18. Evenson KR, Sarmiento OL, Ayala GX. Acculturation and physical activity among North Carolina Latina immigrants. Soc Sci Med 2004 Dec;59(12):2509-2522. [doi: 10.1016/j.socscimed.2004.04.011] [Medline: 15474205]

19. Perez LG, Chavez A, Marquez DX, Soto SC, Haughton J, Arredondo EM. Associations of Acculturation With Self-Report and Objective Physical Activity and Sedentary Behaviors Among Latinas. Health Educ Behav 2017 Jun;44(3):431-438 [FREE Full text] [doi: 10.1177/1090198116669802] [Medline: 27679665]

20. Nicaise V, Marshall S, Ainsworth BE. Domain-specific physical activity and self-report bias among low-income Latinas living in San Diego County. J Phys Act Health 2011 Sep;8(7):881-890. [doi: 10.1123/jpah.8.7.881] [Medline: 21885878]

21. Holtermann A, Hansen JV, Burr H, Søgaard K, Sjøgaard G. The health paradox of occupational and leisure-time physical activity. Br J Sports Med 2012 Mar;46(4):291-295. [doi: 10.1136/bjsm.2010.079582] [Medline: 21459873]

22. Samitz G, Egger M, Zwahlen M. Domains of physical activity and all-cause mortality: systematic review and dose-response meta-analysis of cohort studies. Int J Epidemiol 2011 Oct;40(5):1382-1400. [doi: 10.1093/ije/dyr112] [Medline: 22039197]

23. Autenrieth CS, Baumert J, Baumeister SE, Fischer B, Peters A, Döring A, et al. Association between domains of physical activity and all-cause, cardiovascular and cancer mortality. Eur J Epidemiol 2011 Feb;26(2):91-99. [doi:

10.1007/s10654-010-9517-6] [Medline: 21153912]

24. Harari G, Green MS, Zelber-Sagi S. Combined association of occupational and leisure-time physical activity with all-cause and coronary heart disease mortality among a cohort of men followed-up for 22 years. Occup Environ Med 2015 Sep;72(9):617-624. [doi: 10.1136/oemed-2014-102613] [Medline: 25805756]

25. Holtermann A, Marott JL, Gyntelberg F, Søgaard K, Mortensen OS, Prescott E, et al. Self-reported occupational physical activity and cardiorespiratory fitness: Importance for cardiovascular disease and all-cause mortality. Scand J Work Environ Health 2016 Jul 01;42(4):291-298 [FREE Full text] [doi: 10.5271/sjweh.3563] [Medline: 27100403]

26. Wanner M, Martin BW, Autenrieth CS, Schaffner E, Meier F, Brombach C, et al. Associations between domains of physical activity, sitting time, and different measures of overweight and obesity. Prev Med Rep 2016 Jun;3:177-184 [FREE Full text] [doi: 10.1016/j.pmedr.2016.01.007] [Medline: 27419012]

27. American Community Survey: 2015-2019 ACS 5-Year Data Profile. United States Census Bureau.: United States Census Bureau; 2015. URL: https://www.census.gov/acs/www/data/data-tables-and-tools/data-profiles/ [accessed 2021-01-28]

28. 2017 Behavioral Risk Factor Surveillance System Questionnaire. Centers for Disease Control and Prevention. URL: https:/ /www.cdc.gov/brfss/questionnaires/pdf-ques/2017 BRFSS Pub Ques 508 tagged.pdf [accessed 2021-04-26]

29. Craig CL, Marshall AL, Sjöström M, Bauman AE, Booth ML, Ainsworth BE, et al. International physical activity questionnaire: 12-country reliability and validity. Med Sci Sports Exerc 2003 Aug;35(8):1381-1395. [doi: 10.1249/01.MSS.0000078924.61453.FB] [Medline: 12900694]

30. Roman-Viñas B, Serra-Majem L, Hagströmer M, Ribas-Barba L, Sjöström M, Segura-Cardona R. International Physical Activity Questionnaire: Reliability and validity in a Spanish population. Eur J Sport Sci 2010 Sep;10(5):297-304. [doi: 10.1080/17461390903426667]

31. The IPAQ Group. Guidelines for data processing and analysis of the International Physical Activity Questionnaire (IPAQ) - Short and Long Forms. International Physical Activity Questionnaire. 2005 Nov. URL: https://docs.google.com/ viewer? $\mathrm{a}=\mathrm{v} \&$ pid=sites\&srcid=ZGVmYXVsdGRvbWFpbnx0aGVpcGFxfGd4OjE0NDgxMDk3NDU1YWRIZTM [accessed 2021-04-26]

32. Mujahid MS, Diez Roux AV, Morenoff JD, Raghunathan T. Assessing the measurement properties of neighborhood scales: from psychometrics to ecometrics. Am J Epidemiol 2007 Apr 15;165(8):858-867. [doi: 10.1093/aje/kwm040] [Medline: $\underline{17329713}$ ]

33. Keller C, Ainsworth B, Records K, Todd M, Belyea M, Vega-López S, et al. A comparison of a social support physical activity intervention in weight management among post-partum Latinas. BMC Public Health 2014 Sep 19;14:971 [FREE Full text] [doi: 10.1186/1471-2458-14-971] [Medline: 25233867]

34. Tovar M, Walker JL, Rew L. Factors Associated With Physical Activity in Latina Women: A Systematic Review. West J Nurs Res 2018 Feb;40(2):270-297. [doi: 10.1177/0193945916681004] [Medline: 27920349] 
35. Smith AD, Crippa A, Woodcock J, Brage S. Physical activity and incident type 2 diabetes mellitus: a systematic review and dose-response meta-analysis of prospective cohort studies. Diabetologia 2016 Dec;59(12):2527-2545 [FREE Full text] [doi: 10.1007/s00125-016-4079-0] [Medline: 27747395]

36. Aune D, Norat T, Leitzmann M, Tonstad S, Vatten LJ. Physical activity and the risk of type 2 diabetes: a systematic review and dose-response meta-analysis. Eur J Epidemiol 2015 Jul;30(7):529-542. [doi: 10.1007/s10654-015-0056-z] [Medline: 26092138]

37. Prince SA, Adamo KB, Hamel ME, Hardt J, Connor Gorber S, Tremblay M. A comparison of direct versus self-report measures for assessing physical activity in adults: a systematic review. Int J Behav Nutr Phys Act 2008 Nov 06;5:56 [FREE Full text] [doi: 10.1186/1479-5868-5-56] [Medline: 18990237$]$

38. Tudor-Locke C, Johnson W, Katzmarzyk P. Frequently reported activities by intensity for U.S. adults: the American Time Use Survey. Am J Prev Med 2010 Oct;39(4):e13-e20. [doi: 10.1016/j.amepre.2010.05.017] [Medline: 20837277]

39. Barnett DW, Barnett A, Nathan A, Van Cauwenberg J, Cerin E, Council on EnvironmentPhysical Activity (CEPA) - Older Adults working group. Built environmental correlates of older adults' total physical activity and walking: a systematic review and meta-analysis. Int J Behav Nutr Phys Act 2017 Aug 07;14(1):103 [FREE Full text] [doi: 10.1186/s12966-017-0558-z] [Medline: 28784183]

40. Silfee VJ, Rosal MC, Sreedhara M, Lora V, Lemon SC. Neighborhood environment correlates of physical activity and sedentary behavior among Latino adults in Massachusetts. BMC Public Health 2016 Sep 13;16:966 [FREE Full text] [doi: 10.1186/s12889-016-3650-4] [Medline: 27619205]

41. Van Holle V, Van Cauwenberg J, Van Dyck D, Deforche B, Van de Weghe N, De Bourdeaudhuij I. Relationship between neighborhood walkability and older adults' physical activity: results from the Belgian Environmental Physical Activity Study in Seniors (BEPAS Seniors). Int J Behav Nutr Phys Act 2014 Aug 23;11:110 [FREE Full text] [doi: 10.1186/s12966-014-0110-3] [Medline: 25148845]

42. Pratt M, Yin S, Soler R, Njai R, Siegel PZ, Liao Y. Does perceived neighborhood walkability and safety mediate the association between education and meeting physical activity guidelines? Prev Chronic Dis 2015 Apr 09;12:E46 [FREE Full text] [doi: 10.5888/pcd12.140570] [Medline: 25855989]

\section{Abbreviations \\ IPAQ: International Physical Activity Questionnaire \\ MET: metabolic equivalent \\ OLS: ordinal least squares \\ PA: physical activity}

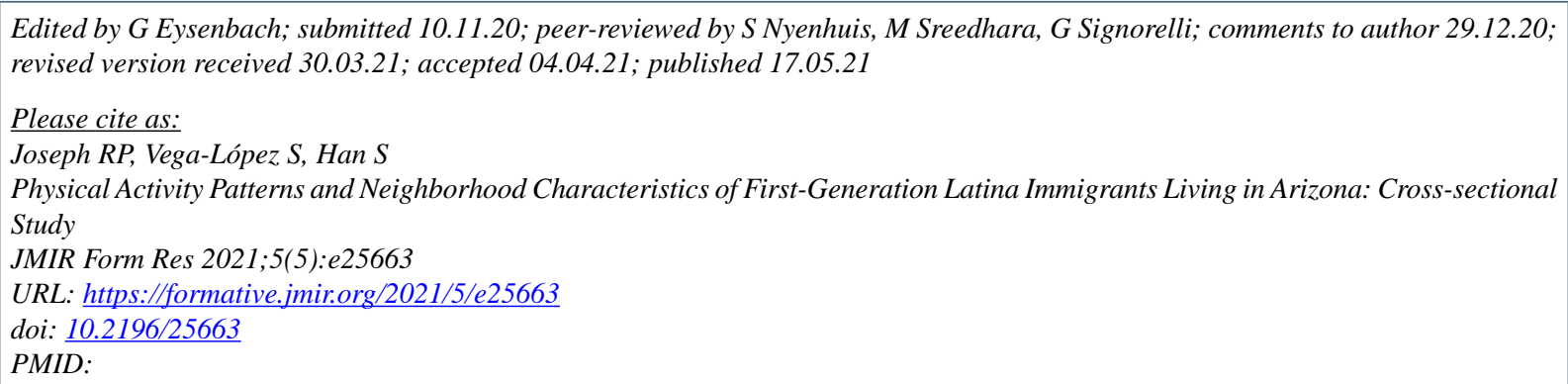

CRodney P Joseph, Sonia Vega-López, SeungYong Han. Originally published in JMIR Formative Research (https://formative.jmir.org), 17.05.2021. This is an open-access article distributed under the terms of the Creative Commons Attribution License (https://creativecommons.org/licenses/by/4.0/), which permits unrestricted use, distribution, and reproduction in any medium, provided the original work, first published in JMIR Formative Research, is properly cited. The complete bibliographic information, a link to the original publication on https://formative.jmir.org, as well as this copyright and license information must be included. 